\title{
Strong convergence theorems for fixed points of asymptotically nonexpansive semigroups in Banach spaces
}

\author{
Hossein Piri ${ }^{1}$ and Poom Kumam ${ }^{2 *}$
}

\section{"Correspondence:}

poom.kum@kmutt.ac.th; poom.kumam@mail.kmutt.ac.th

${ }^{2}$ Department of Mathematics,

Faculty of Science, King Mongkut's University of Technology Thonburi (KMUTT), 126 Bang Mod, Thrung Khru, Bangkok, 10140, Thailand Full list of author information is available at the end of the article

\begin{abstract}
The purpose of this paper is to study the viscosity iterative schemes for approximating a fixed point of an asymptotically nonexpansive semigroup on a compact convex subset of a smooth Banach space with respect to a sequence $\left\{\mu_{i, n}\right\}_{i=1, n=1}^{m, \infty}$ of strongly asymptotic invariant means defined on an appropriate space of bounded real valued functions of the semigroup. Our results extend and improve the result announced by Lau et al. (Nonlinear Anal. 67(4):1211-1225, 2007) and many others.
\end{abstract}

\section{Introduction}

Let $E^{*}$ be the topological dual of a real Banach space $E$ and $C$ be a nonempty closed and convex subset of $E$. The value of $j \in E^{*}$ at $x \in E$ will be denoted by $\langle x, j\rangle$ or $j(x)$. With each $x \in E$, we associate the set

$$
J(x)=\left\{j \in E^{*}:\langle x, j\rangle=\|x\|^{2}=\|j\|^{2}\right\} .
$$

Using the Hahn-Banach theorem, it is immediately clear that $J(x) \neq \phi$ for each $x \in E$. The multi-valued mapping $J$ from $E$ into $E^{*}$ is said to be the (normalized) duality mapping. Let $U=\{x \in E:\|x\|=1\}$. A Banach space $E$ is said to be uniformly convex, if for any $\epsilon \in(0,2]$, there exists a $\delta>0$ such that, for any $x, y \in U,\|x-y\| \geq 0$ implies $\left\|\frac{x+y}{2}\right\| \leq 1-\delta$. It is well known that a uniformly convex Banach space is reflexive and strictly convex. A Banach space $E$ is said to be smooth if the $\operatorname{limit}_{t \rightarrow 0} \frac{\|x+t y\|-\|x\|}{t}$ exists for all $x, y \in U$. As is well known, the duality mapping is norm to weak-star continuous when $E$ is smooth; see [1]. Recall that a mapping $T$ of $C$ into itself is said to be:

(1) Lipschitzian with Lipschiz constant $l>0$ if

$$
\|T x-T y\| \leq l\|x-y\|, \quad \forall x, y \in C,
$$

(2) nonexpansive if

$$
\|T x-T y\| \leq\|x-y\|, \quad \forall x, y \in C
$$

\section{Springer}

@2014 Piri and Kumam; licensee Springer. This is an Open Access article distributed under the terms of the Creative Commons Attribution License (http://creativecommons.org/licenses/by/2.0), which permits unrestricted use, distribution, and reproduction in any medium, provided the original work is properly cited. 
(3) asymptotically nonexpansive if there exists a sequence $\left\{l_{n}\right\}$ of positive numbers such that $\lim _{n \rightarrow \infty} l_{n}=1$ and

$$
\left\|T^{n} x-T^{n} y\right\| \leq l_{n}\|x-y\|, \quad \forall x, y \in C
$$

Iteration processes are often used to approximate a fixed point of a nonexpansive mapping $T$. The first one is introduced by Halpern [2] and is defined as follows: Take an initial guess $x=x_{0} \in C$ arbitrarily and define $x_{n}$ recursively by

$$
x_{n+1}=\alpha_{n} x_{0}+\left(1-\alpha_{n}\right) T x_{n}, \quad n \geq 0
$$

where $\left\{\alpha_{n}\right\}$ is a sequence in $[0,1]$.

In 2007, Lau et al. [3] introduced Halpern's iterative schemes for approximating fixed point of semigroup $\varphi=\{T(s): s \in S\}$ of nonexpansive mappings on a nonempty compact convex subset $C$ of Smooth (and strictly convex) Banach space and introduced the following iteration process. Let $x=x_{0} \in C$ and

$$
x_{n+1}=\alpha_{n} x+\left(1-\alpha_{n}\right) T_{\mu_{n}} x_{n}, \quad n \geq 1,
$$

where $\left\{\mu_{n}\right\}_{n=1}^{\infty}$ is a sequence of left strong regular invariant means defined on an appropriate invariant subspace of $l^{\infty}(S)$.

A semigroup $S$ is called left reversible if any two right ideals of $S$ have nonvoid intersection, i.e., $a S \cap b S \neq \emptyset$ for $a, b \in S$. In this case, $(S, \preceq)$ is a directed set when the binary relation $\preceq$ on $S$ is defined by $a \preceq b$ if and only if $a S \supset b S$ for $a, b \in S . \varphi=\{T(s): s \in S\}$ is called a Lipschitzian semigroup on $C$ if $T(s)$ be a Lipschitzian mapping of $C$ into $C$ with Lipschitz constant $l(s)$ for each $s \in S, T(s t)=T(s) T(t)$ for each $t, s \in S$ and $T(e)=I$. A Lipschitzian semigroup $\varphi=\{T(s): s \in S\}$ is called nonexpansive (or a contractive) semigroup if $l(s)=1$, for each $s \in S$, and asymptotically nonexpansive semigroup if $\lim _{s} l(s) \leq 1$. Left revisable semigroup of nonexpansive mappings were first studied by Lau [4] and Holmes and Lau [5].

In this paper, motivated and inspired by Lau et al. [3], Katchang and Kumam [6], Kumam et al. [7], Razani and Yazdi [8], Piri [9], Piri and Badali [10], Piri and Kumam [11], Piri et al. [12], Saewan and Kumam [13], we introduce the composite explicit viscosity iterative schemes as follows:

$$
\begin{aligned}
& y_{m+1, n}=x_{n}, \\
& y_{i, n}=\delta_{i, n} y_{i+1, n}+\left(I-\delta_{i, n}\right) T\left(\mu_{i, n}\right) y_{i+1, n}, \quad i=1,2, \ldots, m, \\
& x_{n+1}=\alpha_{n} \gamma f\left(x_{n}\right)+\beta_{n} x_{n}+\left(\left(1-\beta_{n}\right) I-\alpha_{n} A\right) T\left(\mu_{i, n}\right) y_{i, n},
\end{aligned}
$$

where $f$ is a weakly contractive mapping and $A$ is a strongly positive bounded linear operator on $E$ with coefficient $\bar{\gamma}>0$ and $0<\gamma<\bar{\gamma}$, for an asymptotically nonexpansive semigroup $\varphi=\{T(s): s \in S\}$ on compact convex subset $C$ of a smooth Banach space $E$ with respect to finite family of left strongly asymptotically invariant sequences $\left\{\mu_{i, n}\right\}_{i=1, n=1}^{m, \infty}$ of means defined on an appropriate invariant subspace of $l^{\infty}(S)$. We prove, under certain ap- 
propriate assumptions on the sequences $\left\{\alpha_{n}\right\}_{n=1}^{\infty},\left\{\beta_{n}\right\}_{n=1}^{\infty}$, and $\left\{\delta_{n}\right\}_{i=1, n=1}^{m, \infty}$, that $\left\{x_{n}\right\}_{n=1}^{\infty}$ and $\left\{y_{n}\right\}_{i=1, n=1}^{m, \infty}$ defined by (3) converges strongly to $z \in \operatorname{Fix}(\varphi)$, which is the unique solution of the variational inequality:

$$
\langle(\gamma f-A) z, J(y-z)\rangle \leq 0, \quad \forall y \in \operatorname{Fix}(\varphi)
$$

Our results improve and extend many previous results of Lau et al. [3], Saeidi [14], Saeidi and Naseri [15], Katchang and Kumam [6] and Piri and Kumam [11] and many others.

\section{Preliminaries}

Let $S$ be a semigroup and let $l^{\infty}(S)$ be the space of all bounded real valued functions defined on $S$ with supremum norm. For $s \in S$ and $f \in l^{\infty}(S)$, we define elements $l(s) f$ and $r(s) f$ in $l^{\infty}(S)$ by

$$
(l(s) f)(t)=f(s t), \quad(r(s) f)(t)=f(t s), \quad \forall t \in S .
$$

Let $X$ be a closed subspace of $l^{\infty}(S)$ containing 1 and let $X^{*}$ be its topological dual. An element $\mu$ of $X^{*}$ is said to be a mean on $X$ if $\|\mu\|=\mu(1)=1$. We often write $\mu_{t}(f(t))$ instead of $\mu(f)$ for $\mu \in X^{*}$ and $f \in X$. Let $X$ be left invariant (resp. right invariant), i.e., $l(s)(X) \subset X$ (resp. $r(s)(X) \subset X$ ) for each $s \in S$. A mean $\mu$ on $X$ is said to be left invariant (resp. right invariant) if $\mu(l(s) f)=\mu(f)$ (resp. $\mu(r(s) f)=\mu(f))$ for each $s \in S$ and $f \in X . X$ is said to be left (resp. right) amenable if $X$ has a left (resp. right) invariant mean. $X$ is amenable if $X$ is both left and right amenable. As is well known, $l^{\infty}(S)$ is amenable when $S$ is a commutative semigroup; see [3]. A net $\left\{\mu_{\alpha}\right\}$ of means on $X$ is said to be left strongly asymptotically if

$$
\lim _{\alpha}\left\|l(s)^{*} \mu_{\alpha}-\mu_{\alpha}\right\|=0
$$

for each $s \in S$, where $l(s)^{*}$ is the adjoint operator of $l(s)$.

Let $C$ be a nonempty closed and convex subset of $E$. Throughout this paper, $S$ will always denote a semigroup with an identity $e . \mathrm{S}$ is called left reversible if any two right ideals in $S$ have nonvoid intersection, that is, $a S \cap b S \neq \emptyset$, for $a, b \in S$. In this case, we can define a partial ordering $\prec$ on $S$ by $a \prec b$ if and only if $a S \supset b S$. It is easy too see $t \prec t s, \forall t, s \in S$. Further, if $t \prec s$ then $p t \prec p s$ for all $p \in S$. If a semigroup $S$ is left amenable, then $S$ is left reversible (see [16]). But the converse is false. $\varphi=\{T(s): s \in S\}$ is called a Lipschitzian semigroup on $C$ if $T(s)$ be a Lipschitzian mapping of $C$ into $C$ with Lipschitz constant $l(s)$ for each $s \in S, T(s t)=T(s) T(t)$ for each $t, s \in S$ and $T(e)=I$. A Lipschitzian semigroup $\varphi=\{T(s): s \in S\}$ is called nonexpansive (or a contractive) semigroup if $l(s)=1$, for each $s \in S$, and asymptotically nonexpansive semigroup if $\lim _{s} l(s) \leq 1$. We denote by $\operatorname{Fix}(\varphi)$ the set of common fixed points of $\varphi$, and by $C_{a}$ the set of almost periodic elements in $C$, that is, all $x \in C$ such that $\{T(s) x: s \in S\}$ is relatively compact in the norm topology of $E$. We will call a subspace $X$ of $l^{\infty}(S), \varphi$-stable if the functions $s \rightarrow\left\langle T(s) x, x^{*}\right\rangle$ and $s \rightarrow\|T(s) x-y\|$ on $S$ are in $X$ for all $x, y \in C$ and $x^{*} \in E^{*}$. We know that if $\mu$ is a mean on $X$ and if for each $x^{*} \in E^{*}$ the function $s \rightarrow\left\langle T(s) x, x^{*}\right\rangle$ is contained in $X$ and $C$ is weakly compact, then there exists a unique point $x_{0}$ of $E$ such that $\mu_{s}\left\langle T(s) x, x^{*}\right\rangle=\left\langle x_{0}, x^{*}\right\rangle$ for each $x^{*} \in E^{*}$. We denote such a point $x_{0}$ by $T(\mu) x$. Note that $T(\mu) z=z$, for each $z \in \operatorname{Fix}(\varphi)$ (see [17]). 
Lemma 2.1 [18] Let $S$ be a left reversible semigroup and $\varphi=\{T(s): s \in S\}$ be an asymptotically nonexpansive semigroup on weakly compact convex subset $C$ of a Banach space E. Let $X$ be a left invariant and $\varphi$-stable subspace of $B(S)$ and $\mu$ be an asymptotically left strongly asymptotically invariant means on $X$. Then $\operatorname{Fix}(\varphi)=\operatorname{Fix}(T(\mu)) \cap C_{a}$.

Lemma 2.2 [14] Let $S$ be a left reversible semigroup and $\varphi=\{T(s): s \in S\}$ be an asymptotically nonexpansive semigroup on weakly compact convex subset $C$ of a Banach space $E$ into C. Let $X$ be a left invariant and $\varphi$-stable subspace of $B(S)$ and $\left\{\mu_{n}\right\}_{n=1}^{\infty}$ be an asymptotically left invariant sequence of means on $X$. If $z \in C_{a}$ and $\liminf _{n \rightarrow \infty}\left\|T\left(\mu_{n}\right) z-z\right\|=0$, then $z$ is a common fixed point of $\varphi$.

Let $D$ be a subset of $B$, where $B$ is a subset of a Banach space $E$ and let $P$ be a retraction of $B$ onto $D$, that is, $P x=x$ for each $x \in D$. Then $P$ is said to be sunny [19] if for each $x \in B$ and $t \geq 0$ with $P x+t(x-P x) \in B, P(P x+t(x-P x))=P x$. A subset $D$ of $B$ is said to be a sunny nonexpansive retract of $B$, if there exists a sunny nonexpansive retraction $P$ of $B$ into $D$.

Lemma 2.3 [14] Let $S$ be a left reversible semigroup and $\varphi=\{T(s): s \in S\}$ be an asymptotically nonexpansive semigroup on a nonempty compact convex subset $C$ of a Banach space $E$ into $C$. Let $X$ be a left invariant and $\varphi$-stable subspace of $L^{\infty}(S)$ and $\mu$ be a left invariant sequence of means on $X$. Then $T(\mu)$ is nonexpansive and $\operatorname{Fix}(\varphi) \neq \emptyset$. Moreover, if $E$ is smooth, then $\operatorname{Fix}(\varphi)$ is a sunny nonexpansive retract of $C$ and the sunny nonexpansive retraction of $C$ onto $C$ onto $\operatorname{Fix}(\varphi)$ is unique.

Lemma 2.4 [1] Let $C$ be a nonempty convex subset of smooth Banach space $E, D$ a nonempty subset of $C$, and $P: C \rightarrow D$ a retraction. Then the following are equivalent:

(a) $P$ is the sunny nonexpansive.

(b) $\langle x-P x, J(y-P x)\rangle \leq 0$ for all $x \in C$ and $y \in D$.

(c) $\langle x-y, J(P x-P y)\rangle \geq\|P x-P y\|^{2}$ for all $x, y \in C$.

In a smooth Banach space, an operator $A$ is strongly positive if there exists a constant $\bar{\gamma}>0$ with the property that

$$
\langle A x, J(x)\rangle \geq \bar{\gamma}\|x\|^{2}, \quad\|a I-b A\|=\sup _{\|x\| \leq 1}|\langle(a I-b A) x, J(x)\rangle|, \quad a \in[0,1], b \in[-1,1],
$$

where $I$ is the identity mapping and $J$ is the normalized duality mapping.

Lemma 2.5 [20] If $A$ is a strongly positive bounded linear operator on a smooth Banach space $E$ with coefficient $\bar{\gamma}>0$ and $0<\rho \leq\|A\|^{-1}$, then $\|I-\rho A\| \leq 1-\rho \bar{\gamma}$.

Definition 2.6 [21] A self-mapping $f: C \rightarrow C$ is called weakly contractive of the class $C_{\psi(s)}$ if there exists a continuous and nondecreasing function $\psi:[0, \infty) \rightarrow[0, \infty)$ such that $\psi(s)>0, \forall s>0, \psi(0)=0, \lim _{s \rightarrow \infty} \psi(s)=\infty$, and for any $x, y \in C$,

$$
\|f(x)-f(y)\| \leq\|x-y\|-\psi(\|x-y\|) .
$$


Remark 2.7 Clearly a contractive mapping with constant $\mathrm{k}$ must be a weakly contractive mapping, where $\psi(s)=(1-k)$, but the converse is not true. For example the mapping $f(x)=$ $\sin (x)$ from $[0,1]$ to $[0,1]$ is weakly contractive with $\psi(s)=\frac{1}{8} s^{3}$. But $f$ is not a contractive mapping (see [22]).

Lemma 2.8 [23] Let $\left\{x_{n}\right\}_{n=1}^{\infty}$ and $\left\{y_{n}\right\}_{n=1}^{\infty}$ be bounded sequences in a Banach space $X$ and let $\left\{\alpha_{n}\right\}_{n=1}^{\infty}$ be a sequence in $[0,1]$ such that $0<\liminf _{n \rightarrow \infty} \alpha_{n} \leq \lim _{\sup _{n \rightarrow \infty}} \alpha_{n}<1$. If $x_{n+1}=$ $\alpha_{n} x_{n}+\left(1-\alpha_{n}\right) y_{n}$ for all integers $n \geq 0$ and

$$
\limsup _{n \rightarrow \infty}\left(\left\|y_{n+1}-y_{n}\right\|-\left\|x_{n+1}-x_{n}\right\|\right) \leq 0
$$

then $\lim _{n \rightarrow \infty}\left\|y_{n}-x_{n}\right\|=0$.

Lemma 2.9 [24] Let E be a real smooth Banach space and J be the duality mapping. Then

$$
\|x+y\|^{2} \leq\|x\|^{2}+2\langle y, J(x+y)\rangle, \quad \forall x, y \in E .
$$

Lemma 2.10 [25] Let $\left\{a_{n}\right\}_{n=1}^{\infty}$ be a sequence of nonnegative real numbers such that

$$
a_{n+1} \leq\left(1-b_{n}\right) a_{n}+c_{n}, \quad n \geq 0,
$$

where $\left\{b_{n}\right\}_{n=1}^{\infty}$ and $\left\{c_{n}\right\}_{n=1}^{\infty}$ are sequences of real numbers satisfying the following conditions:

(i) $\left\{b_{n}\right\}_{n=1}^{\infty} \subset(0,1), \sum_{n=0}^{\infty} b_{n}=\infty$,

(ii) either $\lim \sup _{n \rightarrow \infty} \frac{c_{n}}{b_{n}} \leq 0$ or $\sum_{n=0}^{\infty}\left|c_{n}\right|<\infty$.

Then $\lim _{n \rightarrow \infty} a_{n}=0$.

Lemma 2.11 [1] Let $(X, d)$ be a metric space. A subset $C$ of $X$ is compact if and only if every sequence in $C$ contains a convergent subsequence with limit in $C$.

\section{The main result}

In this section, we establish a strong convergence theorem for finding a common fixed point of an asymptotically nonexpansive semigroup in a smooth Banach space.

Theorem 3.1 Let $S$ be a left reversible semigroup, and let $\varphi=\{T(s): s \in S\}$ be an asymptotically nonexpansive semigroup on a nonempty compact convex subset $C$ of a smooth Banach space $E$ such that $\operatorname{Fix}(\varphi) \neq \emptyset$. Let $f$ be a weakly contractive mapping of the class $C_{\psi(s)}$, and let $A$ be a strongly positive linear operator on $E$ with coefficient $\bar{\gamma}>0$. Let $\gamma$ be a real number such that $0<\gamma<\bar{\gamma}$, and let $X$ be a left amenable and $\varphi$-stable subspace of $L^{\infty}(S)$ containing 1 and the function $t \rightarrow\langle T(t) x, y\rangle$ is an element of $X$ for each $x \in C$ and $y \in H$. Let $\left\{\mu_{i, n}\right\}_{i=1, n=1}^{m, \infty}$ be a finite family of left strongly asymptotically invariant sequence of mean on $X$ such that for $i=1,2, \ldots, m, \lim _{n \rightarrow \infty}\left\|\mu_{i, n+1}-\mu_{i, n}\right\|=0$, and let $\left\{\alpha_{n}\right\}_{n=1}^{\infty}$ be a sequence in $(0,1),\left\{\beta_{n}\right\}_{n=1}^{\infty}$ be a sequence in $[0,1)$ and $\left\{\delta_{n}\right\}_{i=1, n=1}^{m, \infty}$ be sequences in $(0,1]$ satisfying in the following conditions:

(B) $\lim _{n \rightarrow \infty} \alpha_{n}=0, \sum_{n=1}^{\infty} \alpha_{n}=\infty$,

$\left(\mathrm{B}_{2}\right) \lim \sup _{n \rightarrow \infty} \beta_{n}<1$,

(B) $\lim _{n \rightarrow \infty} \delta_{i, n}=1, i=1,2, \ldots, m$. 
If $\left\{x_{n}\right\}_{n=1}^{\infty}$ and $\left\{y_{i, n}\right\}_{i=1, n=1}^{m, \infty}$ are sequences generated by $x_{1} \in C$ and

$$
\begin{aligned}
& y_{m+1, n}=x_{n}, \\
& y_{i, n}=\delta_{i, n} y_{i+1, n}+\left(I-\delta_{i, n}\right) T\left(\mu_{i, n}\right) y_{i+1, n}, \quad i=1,2, \ldots, m, \\
& x_{n+1}=\alpha_{n} \gamma f\left(x_{n}\right)+\beta_{n} x_{n}+\left(\left(1-\beta_{n}\right) I-\alpha_{n} A\right) T\left(\mu_{i, n}\right) y_{i, n},
\end{aligned}
$$

then $\left\{x_{n}\right\}_{n=1}^{\infty}$ and $\left\{y_{i, n}\right\}_{i=1, n=1}^{m, \infty}$ converge strongly to $z \in \operatorname{Fix}(\varphi)$, which is the unique solution of the variational inequality

$$
\langle(\gamma f-A) z, J(y-z)\rangle \leq 0, \quad \forall y \in \operatorname{Fix}(\varphi)
$$

Equivalently, $z=P(\gamma f+(I-A)) z$, where $P$ denotes the unique sunny nonexpansive retraction of $C$ onto $\operatorname{Fix}(\varphi)$.

Proof Since $C$ is a compact convex subset of a Banach space $E$ from Lemma 2.1, we have

$$
\operatorname{Fix}(\varphi)=\bigcap_{i=1}^{m} \operatorname{Fix}\left(T\left(\mu_{i, n}\right)\right) \text {. }
$$

From Lemma 2.3 and definition of $\left\{y_{i, n}\right\}_{i=1, n=1}^{m, \infty}$, for every $z \in \operatorname{Fix}(\varphi)$, we have

$$
\begin{aligned}
\left\|y_{i, n}-z\right\| & =\left\|\delta_{i, n} y_{i+1, n}+\left(1-\delta_{i, n}\right) T\left(\mu_{i, n}\right) y_{i+1, n}-z\right\| \\
& \leq \delta_{i, n}\left\|y_{i+1, n}-z\right\|+\left(1-\delta_{i, n}\right)\left\|T\left(\mu_{i, n}\right) y_{i+1, n}-T\left(\mu_{i, n}\right) z\right\| \\
& =\delta_{i, n}\left\|y_{i+1, n}-z\right\|+\left(1-\delta_{i, n}\right)\left\|y_{i+1, n}-z\right\|=\left\|y_{i+1, n}-z\right\| .
\end{aligned}
$$

Therefore

$$
\left\|y_{1, n}-z\right\| \leq\left\|y_{2, n}-z\right\| \leq \cdots \leq\left\|y_{m, n}-z\right\| \leq\left\|x_{n}-z\right\| .
$$

Since $C$ is compact, it is bounded. So we assume that

$$
M=\sup _{x \in C}\|x\|
$$

First, we show that for any sequence $\left\{u_{n}\right\} \subset C$,

$$
\lim _{n \rightarrow \infty}\left\|T\left(\mu_{i, n+1}\right) u_{n}-T\left(\mu_{i, n}\right) u_{n}\right\|=0, \quad i=1,2, \ldots, m
$$

We have

$$
\begin{aligned}
& \left\|T\left(\mu_{i, n+1}\right) u_{n}-T\left(\mu_{i, n} u_{n}\right)\right\| \\
& \quad=\sup _{x^{*} \in E^{*},\left\|x^{*}\right\|=1}\left|\left\langle T\left(\mu_{i, n+1}\right) u_{n}-T\left(\mu_{i, n}\right) u_{n}, x^{*}\right\rangle\right| \\
& \left.=\sup _{x^{*} \in E^{*},\left\|x^{*}\right\|=1}\left|\left(\mu_{i, n+1}\right)_{s}\left\langle T(s) u_{n}, x^{*}\right\rangle-\left(\mu_{i, n}\right)_{s}\right| T(s) u_{n}, x^{*}\right\rangle \mid
\end{aligned}
$$




$$
\begin{aligned}
& \leq \sup _{x^{*} \in E^{*},\left\|x^{*}\right\|=1}\left\|\mu_{i, n+1}-\mu_{i, n}\right\|\left\|T(s) u_{n}\right\|\left\|x^{*}\right\| \\
& \leq\left\|\mu_{i, n+1}-\mu_{i, n}\right\| M .
\end{aligned}
$$

Since for $i=1,2, \ldots, m, \lim _{n \rightarrow \infty}\left\|\mu_{i, n+1}-\mu_{i, n}\right\|=0$. So, we get (7). Next, we claim that $\lim _{n \rightarrow \infty}\left\|x_{n+1}-x_{n}\right\|=0$. For $z \in \operatorname{Fix}(\varphi)$, from the definition of $\left\{y_{i, n}\right\}_{i=1, n=1}^{m, \infty}$ and Lemma 2.3, we have

$$
\begin{aligned}
&\left\|y_{i, n+1}-y_{i, n}\right\| \\
&=\left\|\delta_{i, n+1} y_{i+1, n+1}+\left(1-\delta_{i, n+1}\right) T\left(\mu_{i, n+1}\right) y_{i+1, n+1}-\delta_{i, n} y_{i+1, n}-\left(1-\delta_{i, n}\right) T\left(\mu_{i, n}\right) y_{i+1, n}\right\| \\
&= \| \delta_{i, n+1} y_{i+1, n+1}-\delta_{i, n+1} y_{i+1, n}+\delta_{i, n+1} y_{i+1, n} \\
&+\left(1-\delta_{i, n+1}\right) T\left(\mu_{i, n+1}\right) y_{i+1, n+1}-\delta_{i, n} y_{i+1, n}-\left(1-\delta_{i, n}\right) T\left(\mu_{i, n}\right) y_{i+1, n} \| \\
& \leq \delta_{i, n+1}\left\|y_{i+1, n+1}-y_{i+1, n}\right\|+\left|\delta_{i, n+1}-\delta_{i, n}\right|\left\|y_{i+1, n}\right\| \\
&+\left(1-\delta_{i, n+1}\right)\left\|T\left(\mu_{i, n+1}\right) y_{i+1, n+1}\right\|+\left(1-\delta_{i, n}\right)\left\|T\left(\mu_{i, n}\right) y_{i+1, n}\right\| \\
& \leq\left\|y_{i+1, n+1}-y_{i+1, n}\right\|+\left|\delta_{i, n+1}-\delta_{i+1, n}\right|\left\|y_{i+1, n}\right\| \\
&+\left(1-\delta_{i, n+1}\right)\left\|T\left(\mu_{i, n+1}\right) y_{i+1, n+1}\right\|+\left(1-\delta_{i, n}\right)\left\|T\left(\mu_{i, n}\right) y_{i+1, n}\right\| \\
& \leq\left\|y_{i+1, n+1}-y_{i+1, n}\right\|+\left|\delta_{i, n+1}-\delta_{i+1, n}\right|\left\|y_{i+1, n}\right\| \\
&+\left(1-\delta_{i, n+1}\right)\left[\left\|T\left(\mu_{i, n+1}\right) y_{i+1, n+1}-z\right\|+\|z\|\right] \\
&+\left(1-\delta_{i, n}\right)\left[\left\|T\left(\mu_{i, n}\right) y_{i+1, n}-z\right\|+\|z\|\right] \\
& \leq\left\|y_{i+1, n+1}-y_{i+1, n}\right\|+\left|\delta_{i, n+1}-\delta_{i+1, n}\right|\left\|y_{i+1, n}\right\| \\
&+\left(1-\delta_{i, n+1}\right)\left[\left\|y_{i+1, n+1}-z\right\|+\|z\|\right]+\left(1-\delta_{i, n}\right)\left[\left\|y_{i+1, n}-z\right\|+\|z\|\right], \\
& \leq\left\|y_{i+1, n+1}-y_{i+1, n}\right\|+\left[\left|\delta_{i, n+1}-\delta_{i+1, n}\right|+\left(1-\delta_{i, n+1}\right)+\left(1-\delta_{i, n}\right)\right] 3 M,
\end{aligned}
$$

which implies that

$$
\left\|y_{i, n+1}-y_{i, n}\right\| \leq\left\|x_{n+1}-x_{n}\right\|+3 M \sum_{j=i}^{m}\left[\left|\delta_{j, n+1}-\delta_{j, n}\right|+2-\left(\delta_{i, n+1}+\delta_{i, n}\right)\right] .
$$

Setting $x_{n+1}=\left(1-\beta_{n}\right) z_{n}+\beta_{n} x_{n}$, we see that $z_{n}=\frac{x_{n+1}-\beta_{n} x_{n}}{1-\beta_{n}}$. Then we compute

$$
\begin{aligned}
z_{n+1}- & z_{n} \\
= & \frac{x_{n+2}-\beta_{n+1} x_{n+1}}{1-\beta_{n+1}}-\frac{x_{n+1}-\beta_{n} x_{n}}{1-\beta_{n}} \\
= & \frac{\alpha_{n+1} \gamma f\left(x_{n+1}\right)+\beta_{n+1} x_{n+1}+\left(\left(1-\beta_{n+1}\right) I-\alpha_{n+1} A\right) T\left(\mu_{i, n+1}\right) y_{i, n+1}-\beta_{n+1} x_{n+1}}{1-\beta_{n+1}} \\
& \quad-\frac{\alpha_{n} \gamma f\left(x_{n}\right)+\beta_{n} x_{n}+\left(\left(1-\beta_{n}\right) I-\alpha_{n} A\right) T\left(\mu_{i, n}\right) y_{i, n}-\beta_{n} x_{n}}{1-\beta_{n}} \\
= & \frac{\alpha_{n+1}}{1-\beta_{n+1}}\left(\gamma f\left(y_{1, n+1}\right)-A T\left(\mu_{i, n+1}\right) y_{i, n+1}\right)-\frac{\alpha_{n}}{1-\beta_{n}}\left(\gamma f\left(y_{1, n}\right)-A T\left(\mu_{i, n}\right) y_{i, n}\right) \\
& +T\left(\mu_{i, n+1}\right) y_{i, n+1}-T_{\mu_{i, n}} y_{i, n} .
\end{aligned}
$$


It follows that

$$
\begin{aligned}
\left\|z_{n+1}-z_{n}\right\| \leq & \left.\frac{\alpha_{n+1}}{1-\beta_{n+1}} \| \gamma f\left(y_{1, n+1}\right)-A T\left(\mu_{i, n+1}\right) y_{i, n+1}\right) \| \\
& +\frac{\alpha_{n}}{1-\beta_{n}}\left\|\gamma f\left(y_{i, n}\right)-A T\left(\mu_{i, n}\right) y_{i, n}\right\| \\
& +\left\|T\left(\mu_{i, n+1}\right) y_{i, n+1}-T_{\mu_{i, n}} y_{i, n}\right\| \\
\leq & \left.\frac{\alpha_{n+1}}{1-\beta_{n+1}} \| \gamma f\left(y_{1, n+1}\right)-A T\left(\mu_{i, n+1}\right) y_{i, n+1}\right) \| \\
& +\frac{\alpha_{n}}{1-\beta_{n}}\left\|\gamma f\left(y_{i, n}\right)-A T\left(\mu_{i, n}\right) y_{i, n}\right\| \\
& +\left\|y_{i, n+1}-y_{i, n}\right\|+\left\|T_{\mu_{i, n+1}} y_{i, n}-T\left(\mu_{i, n}\right) y_{i, n}\right\| \\
\leq & \left\|y_{i, n+1}-y_{i, n}\right\|+\left\|T_{\mu_{i, n+1}} y_{i, n}-T\left(\mu_{i, n}\right) y_{i, n}\right\| \\
& +\left(\frac{\alpha_{n+1}}{1-\beta_{n+1}}+\frac{\alpha_{n}}{1-\beta_{n}}\right) 2 M .
\end{aligned}
$$

Therefore, we observe that

$$
\begin{aligned}
& \left\|z_{n+1}-z_{n}\right\|-\left\|x_{n+1}-x_{n}\right\| \\
& \leq 3 M \sum_{j=i}^{m}\left(\left|\delta_{j, n+1}-\delta_{j, n}\right|+2-\left(\delta_{i, n+1}+\delta_{i, n}\right)\right) \\
& \quad+\left\|T_{\mu_{i, n+1}} y_{i, n}-T\left(\mu_{i, n}\right) y_{i, n}\right\|+\left(\frac{\alpha_{n+1}}{1-\beta_{n+1}}+\frac{\alpha_{n}}{1-\beta_{n}}\right) 2 M .
\end{aligned}
$$

So from (7), $\left(B_{1}\right)$, and $\left(B_{2}\right)$, we obtain

$$
\limsup _{n \rightarrow \infty}\left(\left\|z_{n+1}-z_{n}\right\|-\left\|x_{n+1}-x_{n}\right\|\right) \leq 0 .
$$

Applying Lemma 2.8, we obtain $\lim _{n \rightarrow \infty}\left\|x_{n}-z_{n}\right\|=0$. We also have $\left\|x_{n+1}-x_{n}\right\|=(1-$ $\left.\beta_{n}\right)\left\|x_{n}-z_{n}\right\|$, therefore, we get

$$
\lim _{n \rightarrow \infty}\left\|x_{n+1}-x_{n}\right\|=0
$$

We note that

$$
\begin{aligned}
\left\|x_{n}-T\left(\mu_{i, n}\right) y_{i, n}\right\| \leq & \left\|x_{n}-x_{n+1}\right\|+\left\|x_{n+1}-T\left(\mu_{i, n}\right) y_{i, n}\right\| \\
= & \left\|x_{n}-x_{n+1}\right\|+\| \alpha_{n} \gamma f\left(x_{n}\right)+\beta_{n} x_{n} \\
& +\left(\left(1-\beta_{n}\right) I-\alpha_{n} A\right) T\left(\mu_{i, n}\right) y_{i, n}-T\left(\mu_{i, n}\right) y_{i, n} \| \\
\leq & \left\|x_{n}-x_{n+1}\right\|+\alpha_{n}\left\|\gamma f\left(x_{n}\right)-A T\left(\mu_{i, n}\right) y_{i, n}\right\| \\
& +\beta_{n}\left\|x_{n}-T\left(\mu_{i, n}\right) y_{i, n}\right\| \\
\leq & \left\|x_{n}-x_{n+1}\right\|+2 M \alpha_{n}+\beta_{n}\left\|x_{n}-T\left(\mu_{i, n}\right) y_{i, n}\right\| .
\end{aligned}
$$


Thus, we have the following:

$$
\left\|x_{n}-T\left(\mu_{i, n}\right) y_{i, n}\right\| \leq \frac{1}{1-\beta_{n}}\left(\left\|x_{n}-x_{n+1}\right\|+2 M \alpha_{n}\right)
$$

By $(8),\left(B_{1}\right)$, and $\left(B_{2}\right)$, we obtain the following:

$$
\lim _{n \rightarrow \infty}\left\|x_{n}-T\left(\mu_{i, n}\right) y_{i, n}\right\|=0, \quad i=1,2, \ldots, m
$$

We consider

$$
\begin{aligned}
\left\|x_{n}-T\left(\mu_{i, n}\right) x_{n}\right\| & \leq\left\|x_{n}-T\left(\mu_{i, n}\right) y_{i, n}\right\|+\left\|T\left(\mu_{i, n}\right) y_{i, n}-T\left(\mu_{i, n}\right) x_{n}\right\| \\
& \leq\left\|x_{n}-T\left(\mu_{i, n}\right) y_{i, n}\right\|+\left\|y_{i, n}-x_{n}\right\| \\
& \leq\left\|x_{n}-T\left(\mu_{i, n}\right) y_{i, n}\right\|+\sum_{j=i}^{m}\left\|y_{j+1, n}-y_{j, n}\right\| \\
& \leq\left\|x_{n}-T\left(\mu_{i, n}\right) y_{i, n}\right\|+\sum_{j=i}^{m}\left(1-\delta_{j, n}\right)\left\|y_{j+1, n}-T\left(\mu_{j, n}\right) y_{j+1, n}\right\| \\
& \leq\left\|x_{n}-T\left(\mu_{i, n}\right) y_{i, n}\right\|+2 M \sum_{j=i}^{m}\left(1-\delta_{j, n}\right) .
\end{aligned}
$$

By $(9)$ and $\left(B_{3}\right)$, we have the following:

$$
\lim _{n \rightarrow \infty}\left\|x_{n}-T\left(\mu_{i, n}\right) x_{n}\right\|=0
$$

Next, we prove that $\omega\left(\left\{x_{n}\right\}_{n=1}^{\infty}\right) \subset \operatorname{Fix}(\varphi)$, where

$$
\omega\left(\left\{x_{n}\right\}_{n=1}^{\infty}\right):=\left\{x \in C:\left\{x_{n_{j}}\right\}_{j=1}^{\infty} \subset\left\{x_{n}\right\}_{n=1}^{\infty}, \lim _{j \rightarrow \infty}\left\|x_{n_{j}}-x\right\|=0\right\} .
$$

From Lemma 2.11, we get $\omega\left(\left\{x_{n}\right\}_{n=1}^{\infty}\right) \neq \emptyset$. Let $x \in \omega\left(\left\{x_{n}\right\}_{n=1}^{\infty}\right)$. Then there exists a subsequence $\left\{x_{n_{j}}\right\}_{j=1}^{\infty}$ of $\left\{x_{n}\right\}_{n=1}^{\infty}$ such that

$$
\lim _{j \rightarrow \infty}\left\|x_{n_{j}}-x\right\|=0
$$

It follows from Lemma 2.3 that

$$
\begin{aligned}
\limsup _{j \rightarrow \infty}\left\|x-T_{\mu_{1, n_{j}}} x\right\| \leq & \limsup _{j \rightarrow \infty}\left(\left\|x-x_{n_{j}}\right\|+\left\|x_{n_{j}}-T\left(\mu_{1, n_{j}}\right) x_{n_{j}}\right\|\right. \\
& \left.+\left\|T\left(\mu_{1, n_{j}}\right) x_{n_{j}}-T\left(\mu_{1, n_{j}}\right) x\right\|\right) \\
\leq & \limsup _{j \rightarrow \infty}\left(2\left\|x-x_{n_{j}}\right\|+\left\|x_{n_{j}}-T\left(\mu_{1, n_{j}}\right) x_{n_{j}}\right\|\right) .
\end{aligned}
$$

Thus, due to (10), (11), and Lemma 2.2, we get $x \in \operatorname{Fix}(\varphi)$. Since $E$ is smooth, from Lemma 2.3 there exists a unique sunny nonexpansive retraction $P$ of $C$ onto $\operatorname{Fix}(\varphi)$. Since 
$A$ is bounded, without loss of generality, we may assume that $\|A\| \leq 1$. So from Lemma 2.5 , we get $\|I-A\| \leq 1-\bar{\gamma}$. Since $A$ is linear and $f$ is a weak contraction, we have

$$
\begin{aligned}
& \|(\gamma f+(I-A)) x-(\gamma f+(I-A)) y\| \\
& \quad \leq \gamma\|f(x)-f(y)\|+\|(I-A)(x-y)\| \\
& \quad \leq \gamma[\|x-y\|-\psi(\|x-y\|)]+(1-\bar{\gamma})\|x-y\| \\
& \quad \leq(1+\gamma-\bar{\gamma})\|x-y\| .
\end{aligned}
$$

Since $1+\gamma-\bar{\gamma}<1, \gamma f+(I-A)$ is a contraction of $C$ into itself, therefore $P(\gamma f+(I-A))$ is contraction. Then the Banach contraction theorem guarantees that $P(\gamma f+(I-A))$ has a unique fixed point $z$. By Lemma 2.4, $z$ is the unique solution of the variational inequality

$$
\langle(\gamma f-A) z, J(y-z)\rangle \leq 0, \quad \forall y \in \operatorname{Fix}(\varphi)
$$

Next, we prove that

$$
\limsup _{n \rightarrow \infty}\left\langle(\gamma f-A) z, J\left(x_{n}-z\right)\right\rangle \leq 0
$$

Indeed, we can choose a subsequence $\left\{x_{n_{k}}\right\}$ of $\left\{x_{n}\right\}$ such that

$$
\limsup _{n \rightarrow \infty}\left\langle(\gamma f-A) z, J\left(x_{n}-z\right)\right\rangle=\lim _{k \rightarrow \infty}\left\langle(\gamma f-A) z, J\left(x_{n_{k}}-z\right)\right\rangle .
$$

Since $C$ is compact, we may assume, with no loss of generality, that $\left\{x_{n_{k}}\right\}$ converges strongly to some $y \in C$. Since $\omega\left(\left\{x_{n}\right\}_{n=1}^{\infty}\right) \subset \operatorname{Fix}(\varphi)$ and duality mapping $J$ is norm to weakstar continuous from (12) and (13), we have

$$
\limsup _{n \rightarrow \infty}\left\langle(\gamma f-A) z, J\left(x_{n}-z\right)\right\rangle \leq 0
$$

Finally, we show that $\left\{x_{n}\right\}_{n=1}^{\infty}$ converges strongly to $z$. Using Lemma 2.3, Lemma 2.9, and (6), we have

$$
\begin{aligned}
&\left\|x_{n+1}-z\right\|^{2} \\
&=\left\|\alpha_{n}\left[\gamma f\left(x_{n}\right)-A z\right]+\beta_{n}\left[x_{n}-z\right]+\left[\left(1-\beta_{n}\right) I-\alpha_{n} A\right]\left(T\left(\mu_{i, n}\right) y_{i, n}-z\right)\right\|^{2} \\
& \leq\left\|\beta_{n}\left[x_{n}-z\right]+\left[\left(1-\beta_{n}\right) I-\alpha_{n} A\right]\left(T\left(\mu_{i, n}\right) y_{i, n}-z\right)\right\|^{2} \\
&+2 \alpha_{n}\left\langle\gamma f\left(x_{n}\right)-A z, j\left(x_{n+1}-z\right)\right\rangle \\
&=\left\|\beta_{n}\left[x_{n}-z\right]+\left(1-\beta_{n}\right)\left[I-\frac{\alpha_{n}}{1-\beta_{n}} A\right]\left(T\left(\mu_{i, n}\right) y_{i, n}-z\right)\right\|^{2} \\
&+2 \alpha_{n}\left\langle\gamma f\left(x_{n}\right)-A z, j\left(x_{n+1}-z\right)\right\rangle \\
& \leq \beta_{n}\left\|x_{n}-z\right\|^{2}+\left(1-\beta_{n}\right)\left[I-\frac{\alpha_{n}}{1-\beta_{n}} A\right]\left\|\left(T\left(\mu_{i, n}\right) y_{i, n}-z\right)\right\|^{2} \\
&+2 \alpha_{n}\left\langle\gamma f\left(x_{n}\right)-A z, j\left(x_{n+1}-z\right)\right\rangle
\end{aligned}
$$




$$
\begin{aligned}
\leq & \beta_{n}\left\|x_{n}-z\right\|^{2}+\left(1-\beta_{n}\right)\left(1-\frac{\alpha_{n}}{1-\beta_{n}} \bar{\gamma}\right)^{2}\left\|T\left(\mu_{i, n}\right) y_{i, n}-z\right\|^{2} \\
& +2 \alpha_{n} \gamma\left\|f\left(x_{n}\right)-f(z)\right\|\left\|j\left(x_{n+1}-z\right)\right\|+2 \alpha_{n}\left\langle\gamma f(z)-A z, j\left(x_{n+1}-z\right)\right\rangle \\
\leq & \beta_{n}\left\|x_{n}-z\right\|^{2}+\frac{1}{1-\beta_{n}}\left(1-\beta_{n}-\alpha_{n} \bar{\gamma}\right)^{2}\left\|T\left(\mu_{i, n}\right) y_{i, n}-z\right\|^{2} \\
& +2 \alpha_{n} \gamma\left(\left\|x_{n}-z\right\|-\psi\left(\left\|x_{n}-z\right\|\right)\right)\left\|x_{n+1}-z\right\|+2 \alpha_{n}\left\langle\gamma f(z)-A z, j\left(x_{n+1}-z\right)\right\rangle \\
\leq & \beta_{n}\left\|x_{n}-z\right\|^{2}+\frac{1}{1-\beta_{n}}\left(1-\beta_{n}-\alpha_{n} \bar{\gamma}\right)^{2}\left\|y_{i, n}-z\right\|^{2} \\
& +2 \alpha_{n} \gamma\left\|x_{n}-z\right\|\left\|x_{n+1}-z\right\|+2 \alpha_{n}\left\langle\gamma f(z)-A z, j\left(x_{n+1}-z\right)\right\rangle \\
\leq & \beta_{n}\left\|x_{n}-z\right\|^{2}+\frac{1}{1-\beta_{n}}\left(1-\beta_{n}-\alpha_{n} \bar{\gamma}\right)^{2}\left\|x_{n}-z\right\|^{2} \\
& +\alpha_{n} \gamma\left[\left\|x_{n}-z\right\|^{2}+\left\|x_{n+1}-z\right\|^{2}\right]+2 \alpha_{n}\left\langle\gamma f(z)-A z, j\left(x_{n+1}-z\right)\right\rangle
\end{aligned}
$$

and consequently,

$$
\begin{aligned}
\left\|x_{n+1}-z\right\|^{2} \leq & \frac{1}{1-\alpha_{n} \gamma}\left[\frac{1}{1-\beta_{n}}\left(1-\beta_{n}-\alpha_{n} \bar{\gamma}\right)^{2}+\beta_{n}+\alpha_{n} \gamma\right]\left\|x_{n}-z\right\|^{2} \\
& +\frac{2 \alpha_{n}}{1-\alpha_{n} \gamma}\left\langle\gamma f(z)-A z, j\left(x_{n+1}-z\right)\right\rangle \\
\leq & {\left[1-\frac{2 \alpha_{n}(\bar{\gamma}-\gamma)}{1-\alpha_{n} \gamma}\right]\left\|x_{n}-z\right\|^{2}+\frac{\alpha_{n}^{2} \bar{\gamma}^{2}}{\left(1-\alpha_{n} \gamma\right)\left(1-\beta_{n}\right)}\left\|x_{n}-z\right\|^{2} } \\
& +\frac{2 \alpha_{n}}{1-\alpha_{n} \gamma}\left\langle\gamma f(z)-A z, j\left(x_{n+1}-z\right)\right\rangle .
\end{aligned}
$$

Then we have

$$
\left\|x_{n+1}-z\right\|^{2} \leq\left(1-b_{n}\right)\left\|x_{n}-z\right\|^{2}+b_{n} c_{n}
$$

where $b_{n}=\frac{2 \alpha_{n}(\bar{\gamma}-\gamma)}{1-\alpha_{n} \gamma}$ and

$$
c_{n}=\frac{\alpha_{n}^{2} \bar{\gamma}^{2}}{\left(1-\alpha_{n} \gamma\right)\left(1-\beta_{n}\right)}\left\|x_{n}-z\right\|^{2}+\frac{2 \alpha_{n}}{1-\alpha_{n} \gamma}\left\langle\gamma f(z)-A z, j\left(x_{n+1}-z\right)\right\rangle .
$$

It follows from condition $\left(B_{1}\right)$ and (14) that

$$
\sum_{n=1}^{\infty} b_{n}=\infty, \quad \limsup _{n \rightarrow \infty} c_{n} \leq 0
$$

Therefore, applying Lemma 2.10 to (15), we see that $\left\{x_{n}\right\}_{n=1}^{\infty}$ converges strongly to $z$ and since for $i=1,2, \ldots, m,\left\|y_{i, n}-z\right\| \leq\left\|x_{n}-z\right\|,\left\{y_{n}\right\}_{i=1, n=1}^{m, \infty}$ converges strongly to $z$. This completes the proof.

\section{Applications}

Theorem 4.1 [14] Let $S$ be a left reversible semigroup and $\varphi=\left\{T_{s}: s \in S\right\}$ be a representation of $S$ as Lipschitzian mapping from nonempty compact convex subset $C$ of a smooth 
Banach space $E$ into $C$, with the uniform Lipschitzian condition $\lim _{s} K(s) \leq 1$ and $g$ be an $\alpha$-contraction on $C$ for some $0<\alpha<1$. Let $X$ be a left invariant $\varphi$-stable subspace of $L^{\infty}(\varphi)$ containing $1,\left\{\mu_{n}\right\}_{n=1}^{\infty}$ be a sequence of left strongly asymptotically invariant means defined on $X$ such that $\lim _{n \rightarrow \infty}\left\|\mu_{n+1}-\mu_{n}\right\|=0$ and $\left\{c_{n}\right\}_{n=1}^{\infty}$ be the sequence defined by

$$
c_{n}=\sup _{x, y \in C}\left(\left\|T_{\mu_{n}} x-T_{\mu_{n}} y\right\|-\|x-y\|\right), \quad n \geq 1 .
$$

Let $\left\{\alpha_{n}\right\}_{n=1}^{\infty},\left\{\beta_{n}\right\}_{n=1}^{\infty}$, and $\left\{\gamma_{n}\right\}_{n=1}^{\infty}$ be sequences in $(0,1)$ such that

$\left(\mathrm{C}_{1}\right) \alpha_{n}+\beta_{n}+\gamma_{n}=1, n \geq 1$,

$\left(C_{2}\right) \lim _{n \rightarrow \infty} \alpha_{n}=0$,

(C) $\sum_{n=1}^{\infty} \alpha_{n}=\infty$,

$\left(C_{4}\right) \quad 0<\liminf _{n \rightarrow \infty} \beta_{n} \leq \lim \sup _{n \rightarrow \infty} \beta_{n}<1$,

$\left(C_{5}\right) \lim \sup _{n \rightarrow \infty} \frac{c_{n}}{\alpha_{n}} \leq 0$.

Let $\left\{x_{n}\right\}_{n=1}^{\infty}$ be the sequence generated by $x_{1} \in C$ and

$$
x_{n+1}=\alpha_{n} g\left(x_{n}\right)+\beta_{n} x_{n}+\gamma_{n} T\left(\mu_{n}\right) x_{n}, \quad n \geq 1 .
$$

Then the sequence $\left\{x_{n}\right\}_{n=1}^{\infty}$ converges strongly to some $z \in \operatorname{Fix}(\varphi)$, the set of common fixed points of $\varphi$, which is the unique solution of the variational inequality

$$
\langle(g-I) z, J(y-z)\rangle \leq 0, \quad \forall y \in \operatorname{Fix}(\varphi) .
$$

Equivalently, one has $z=P f z$, where $P$ is the unique sunny nonexpansive retraction of $C$ onto $F(\varphi)$.

Proof It is sufficient to take $g=\frac{1}{\gamma} f, A=I, \delta_{i, n}=1$, for $i=1,2, \ldots, m$ and $\gamma_{n}=1-\alpha_{n}-\beta_{n}$ in Theorem 3.1.

Theorem 4.2 [11] Let $\varphi=\{T(s): s \in S\}$ be a representation of $S$ as a Lipschitzian mappings from a nonempty compact convex subset $C$ of a smooth Banach space $E$ into $C$, with the uniform Lipschitzian constant $\lim _{s} l(s) \leq 1$ on the Lipschiz constant of mappings, such that Fix $(\varphi) \neq \emptyset$, and $g$ be a contraction of $C$ into itself with constant $\alpha \in(0,1)$. Let $X$ be a left invariant and $\varphi$-stable subspace of $B(S)$ containing 1 and the function $t \rightarrow\left\langle T_{t} x, y\right\rangle$ is an element of $X$ for each $x \in C$ and $y \in H$ and $\left\{\mu_{i, n}\right\}_{i=1, n=1}^{m, \infty}$ be a finite family of left strongly asymptotically invariant means on $X$ such that for $i=1,2, \ldots, m, \lim _{n \rightarrow \infty}\left\|\mu_{i, n+1}-\mu_{i, n}\right\|=0$. Let $\left\{\alpha_{n}\right\}_{n=1}^{\infty},\left\{\beta_{n}\right\}_{n=1}^{\infty}$ and $\left\{\gamma_{n}\right\}_{n=1}^{\infty}$ be sequences in $(0,1)$ satisfy in conditions $\left(C_{1}\right)-\left(C_{4}\right)$ and $\left\{\delta_{n}\right\}_{i=1, n=1}^{m, \infty}$ be a sequence in $(0,1]$ satisfies in condition

$\left(C_{5}^{\prime}\right) \lim _{n \rightarrow \infty} \delta_{i, n}=1, i=1,2, \ldots, m$.

If $\left\{x_{n}\right\}_{n=1}^{\infty}$ and $\left\{y_{i, n}\right\}_{i=1, n=1}^{m, \infty}$ are sequences generated by $x_{1} \in C$ and

$$
\begin{aligned}
& x_{n+1}=\alpha_{n} g\left(y_{1, n}\right)+\beta_{n} x_{n}+\gamma_{n} T\left(\mu_{1, n}\right) y_{1, n}, \\
& y_{i, n}=\delta_{i, n} y_{i+1, n}+\left(I-\delta_{i, n}\right) T\left(\mu_{i, n}\right) y_{i+1, n}, \quad i=1,2, \ldots, m, \\
& y_{m+1, n}=x_{n},
\end{aligned}
$$


then $\left\{x_{n}\right\}_{n=1}^{\infty}$ and $\left\{y_{i, n}\right\}_{i=1, n=1}^{m, \infty}$ converge strongly to $z \in \operatorname{Fix}(\varphi)$ which is the unique solution of the variational inequality

$$
\langle(g-I) z, J(y-z)\rangle \leq 0, \quad \forall y \in \operatorname{Fix}(\varphi) .
$$

Equivalently, $z=\operatorname{Pg}(z)$, where $P$ denotes the unique sunny nonexpansive retraction of $C$ onto $\operatorname{Fix}(\varphi)$.

Proof It is sufficient to take $g=\frac{1}{\gamma} f, A=I$, and $\gamma_{n}=1-\alpha_{n}-\beta_{n}$ in Theorem 3.1.

Theorem 4.3 [6] Let $S$ be a left reversible semigroup and $\varphi=\left\{T_{s}: s \in S\right\}$ be a representation of $S$ as Lipschitzian mapping from nonempty compact convex subset $C$ of a smooth Banach space E into $C$, with the uniform Lipschitzian condition $\lim _{s} K(s) \leq 1$ and $g$ be an $\alpha$-contraction on $C$ for some $0<\alpha<1$. Let $X$ be a left invariant $\varphi$-stable subspace of $L^{\infty}(\varphi)$ containing $1,\left\{\mu_{n}\right\}_{n=1}^{\infty}$ is a sequence of left strong regular invariant means defined on $X$ such that $\lim _{n \rightarrow \infty}\left\|\mu_{n+1}-\mu_{n}\right\|=0$ and $\left\{c_{n}\right\}_{n=1}^{\infty}$ be the sequence defined by

$$
c_{n}=\sup _{x, y \in C}\left(\left\|T_{\mu_{n}} x-T_{\mu_{n}} y\right\|-\|x-y\|\right), \quad n \geq 1 .
$$

Let $\left\{\alpha_{n}\right\}_{n=1}^{\infty},\left\{\beta_{n}\right\}_{n=1}^{\infty},\left\{\gamma_{n}\right\}_{n=1}^{\infty}$, and $\left\{\delta_{n}\right\}_{n=1}^{\infty}$ be sequences in $(0,1)$ such that

$\left(\mathrm{C}_{1}\right) \alpha_{n}+\beta_{n}+\gamma_{n}=1, n \geq 1$,

(C2) $\lim _{n \rightarrow \infty} \alpha_{n}=0$,

(C) $\sum_{n=1}^{\infty} \alpha_{n}=\infty$,

$\left(C_{4}\right) \quad 0<\liminf _{n \rightarrow \infty} \beta_{n} \leq \lim \sup _{n \rightarrow \infty} \beta_{n}<1$,

(C) $\lim \sup _{n \rightarrow \infty} \frac{c_{n}}{\alpha_{n}} \leq 0$,

(C6) $\lim _{n \rightarrow \infty} \delta_{n}=0$.

Let $\left\{x_{n}\right\}_{n=1}^{\infty}$ be the sequence generated by $x_{1} \in C$ and

$$
\left\{\begin{array}{l}
y_{n}=\delta_{n} x_{n}+\left(1-\delta_{n}\right) T_{\mu_{n}} x_{n} \\
x_{n+1}=\alpha_{n} g\left(x_{n}\right)+\beta_{n} x_{n}+\gamma_{n} y_{n}, \quad n \geq 1 .
\end{array}\right.
$$

Then the sequence $\left\{x_{n}\right\}_{n=1}^{\infty}$ converges strongly to some $z \in \operatorname{Fix}(\varphi)$, which is the unique solution of the variational inequality.

$$
\langle(f-I) z, J(y-z)\rangle \leq 0, \quad \forall y \in F(\varphi) .
$$

Equivalently, one has $z=P f z$, where $P$ is the unique sunny nonexpansive retraction of $C$ onto $F(\varphi)$.

Proof It is sufficient to take $g=\frac{1}{\gamma} f, A=I, \gamma_{n}=1-\alpha_{n}-\beta_{n}$ for all $n \in \mathbb{N}$ and $\delta_{i, n}=1$ for $i=1,2, \ldots, m-1$ in Theorem 3.1.

Theorem 4.4 [3] Let $S$ be a left reversible semigroup and $\varphi=\left\{T_{s}: s \in S\right\}$ be a representation of $S$ as nonexpansive mappings from a compact convex subset $C$ of a strictly convex and smooth Banach space E into $C$ such that $\operatorname{Fix}(\varphi) \neq \emptyset$, let $X$ be an amenable and S-stable subspace of $L^{\infty}(\varphi)$ and let $\left\{\mu_{n}\right\}_{n=1}^{\infty}$ be a strongly left regular sequence of means on $X$. Let $\left\{\alpha_{n}\right\}_{n=1}^{\infty}$ 
be a sequence in $[0,1]$ such that $\lim _{n \rightarrow \infty} \alpha_{n}=0$ and $\sum_{n=1}^{\infty} \alpha_{n}=\infty$. Let $x-1=x \in C$ and let $\left\{x_{n}\right\}_{n=1}^{\infty}$ be the sequence defined by

$$
x_{n+1}=\alpha_{n} x+\left(1-\alpha_{n}\right) T\left(\mu_{n}\right) x_{n}, \quad n=1,2, \ldots
$$

Then $\left\{x_{n}\right\}_{n=1}^{\infty}$ converges strongly to Px, where $P$ denotes the unique sunny nonexpansive retraction of $C$ onto $F(\varphi)$.

Proof It is sufficient to take $f(x)=\frac{1}{\gamma} x, A=I, \beta_{n}=0$ for all $n \in \mathbb{N}$ and $\delta_{i, n}=1$ for $i=1,2, \ldots, m$ in Theorem 3.1.

Theorem 4.5 [15] Let $\varphi=\left\{T_{s}: s \in S\right\}$ be a nonexpansive semigroup on a Hilbert space $H$ such that $\operatorname{Fix}(\varphi) \neq \emptyset$. Let $X$ be a left invariant subspace of $L^{\infty}(\varphi)$ such that $1 \in X$, and the function $t \rightarrow\langle T(t) x, y\rangle$ is an element of $X$ for each $x, y \in H$. Let $\left\{\mu_{n}\right\}_{n=1}^{\infty}$ be a left regular sequence of means on $X$ and let $\left\{\alpha_{n}\right\}_{n=1}^{\infty}$ be a sequence in $[0,1]$ such that $\lim _{n \rightarrow \infty} \alpha_{n}=0$ and $\sum_{n=1}^{\infty} \alpha_{n}=\infty$. Let $A$ be a strongly positive linear bounded operator on $H$ with coefficient $\bar{\gamma}>0$ and $f$ be an $\alpha$-contraction on $H$ for some $0<\alpha<1$. Let $x_{0} \in H$ and let $\left\{x_{n}\right\}_{n=1}^{\infty}$ be generated by $x_{0}$ and

$$
x_{n+1}=\alpha_{n} \gamma f\left(x_{n}\right)+\left(1-\alpha_{n} A\right) T_{\mu_{n}} x_{n} .
$$

Then the sequence $\left\{x_{n}\right\}_{n=1}^{\infty}$ converges strongly to some $z \in \operatorname{Fix}(\varphi)$, the set of common fixed points of $\varphi$, which is the unique solution of the variational inequality

$$
\langle(A-\gamma g) z, y-z\rangle \leq 0, \quad \forall y \in \operatorname{Fix}(\varphi) .
$$

Equivalently, one has $z=P_{\mathrm{Fix}(\varphi)}(I-A+\gamma g) z$.

Proof It is sufficient to take $\beta_{n}=0$ for all $n \in \mathbb{N}$ and $\delta_{i, n}=1$ for $i=1,2, \ldots, m$, in Theorem 3.1.

Remark 4.6 Theorem 3.1 improves and extends Theorem 3.1 of [14], Theorem 3.1 of [6], Theorem 4.1 of [3] and Theorem 3.1 of [15] in the following aspects.

(1) Theorem 3.1 extends the theorem and Theorem 3.1 of [14] forms one sequence of means to a finite family of sequences of means and gives all consequences of this theorem without assumption $\left(\mathrm{C}_{5}\right)$ used in its proof.

(2) Theorem 3.1 extends the theorem and Theorem 3.1 of [6] forms one sequence of means to a finite family of sequences of means and gives all consequences of this theorem without assumption $\left(\mathrm{C}_{5}\right)$ used in its proof.

(3) Theorem 3.1 extends the theorem and Theorem 4.1 of [3] forms one sequence of means to a finite family of sequence of means and gives all consequences of this theorem without the assumption of strict convexity of Banach spaces used in its proof.

(4) Theorem 3.1 extends the theorem and Theorem 3.1 of [15] forms one sequence of means to a finite family of sequence of means and gives all consequences of this theorem from Hilbert spaces to Banach spaces. 


\section{Competing interests}

The authors declare that they have no competing interests.

\section{Authors' contributions}

All authors contributed equally and significantly in writing this paper. All authors read and approved the final manuscript.

\section{Author details}

'Department of Mathematics, Faculty of Basic Science, University of Bonab, P.O. Box 5551-761167, Bonab, Iran.

${ }^{2}$ Department of Mathematics, Faculty of Science, King Mongkut's University of Technology Thonburi (KMUTT), 126 Bang Mod, Thrung Khru, Bangkok, 10140, Thailand.

\section{Acknowledgements}

The second author was supported by the Thailand Research Fund and the King Mongkut's University of Technology Thonburi (Grant No. RSA5780059)

Received: 14 July 2014 Accepted: 16 October 2014 Published: 31 Oct 2014

\section{References}

1. Agarwal, RP, O'Regan, D, Sahu, DR: Fixed Point Theory for Lipschitzian-Type Mappings with Applications. Topological Fixed Point Theory and Its Applications, vol. 6. Springer, New York (2009)

2. Halpern, B: Fixed points of nonexpanding maps. Bull. Am. Math. Soc. 73(6), 957-961 (1967)

3. Lau, AT, Miyake, H, Takahashi, W: Approximation of fixed points for amenable semigroups of nonexpansive mappings in Banach spaces. Nonlinear Anal. 67(4), 1211-1225 (2007)

4. Lau, AT: Invariant means on almost periodic functions and fixed point properties. Rocky Mt. J. Math. 3, 69-76 (1973)

5. Holmes, RD, Lau, AT: Asymptotically non-expansive actions of topological semigroups and fixed points. Bull. Lond. Math. Soc. 3, 343-347 (1971)

6. Katchang, P, Kumam, P: A composite explicit iterative process with a viscosity method for Lipschitzian semigroup in smooth Banach space. Bull. Iran. Math. Soc. 37, 143-159 (2011)

7. Kumam, P, Plubtieng, S, Katchang, P: Viscosity approximation to a common solution of variational inequality problems and fixed point problems for Lipschitzian semigroup in Banach spaces. Math. Sci. 7, 28 (2013). doi:10.1186/2251-7456-7-28

8. Razani, A, Yazdi, M: An iterative method for family of nonexpansive mappings. Math. Rep. 16(66)(1), 7-23 (2014)

9. Piri, H: Hybrid pseudo-viscosity approximation schemes for systems of equilibrium problems and fixed point problems of infinite family and semigroup of non-expansive mappings. Nonlinear Anal. 74, 6788-6804 (2011)

10. Piri, $\mathrm{H}$, Badali, $\mathrm{AH}$ : Strong convergence theorem for amenable semigroups of nonexpansive mappings and variational inequalities. Fixed Point Theory Appl. 2011, Article ID 55 (2011)

11. Piri, H, Kumam, P: Approximating fixed points for a reversible semigroup of Lipschitzian mappings in a smooth Banach space. J. Inequal. Appl. 2013, Article ID 555 (2013)

12. Piri, H, Kumam, P, Sitthithakerngkiet, K: Approximating fixed points for Lipschitzian semigroup and infinite family of nonexpansive mappings with the Meir-Keeler type contraction in Banach spaces. Dyn. Contin. Discrete Impuls. Syst. 21, 201-229 (2014)

13. Saewan, S, Kumam, P: Explicit iterations for Lipschitzian semigroups with the Meir-Keeler type contraction in Banach spaces. J. Inequal. Appl. 2012, Article ID 279 (2012). doi:10.1186/1029-242X-2012-279

14. Saeidi, S: Approximating common fixed points of Lipschitzian semigroup in smooth Banach spaces. Fixed Point Theory Appl. 2008, Article ID 363257 (2008)

15. Saeidi, S, Naseri, S: Iterative methods for semigroups of nonexpansive mappings and variational inequalities. Math. Rep. 12(62)(1), 59-70 (2010)

16. Lau, AT, Zhang, Y: Fixed point properties of semigroups of non-expansive mappings. J. Funct. Anal. 254(10), 2534-2554 (2008)

17. Hirano, N, Kido, K, Takahashi, W: Nonexpansive retractions and nonlinear ergodic theorems in Banach spaces. Nonlinear Anal. 28, 1269-1281 (1988)

18. Saeidi, S: Strong convergence of Browder's type iterations for left amenable semigroups of Lipschitzian mappings in Banach spaces. J. Fixed Point Theory 5, 93-103 (2009)

19. Reich, S: Asymptotic behavior of contraction in Banach spaces. J. Math. Anal. Appl. 44, 57-70 (1973)

20. Cai, G, Hu, CS: Strong convergence theorems of a general iterative process for a finite family of $\lambda_{j}$-strict pseudo-contractions in q-uniformly smooth Banach spaces. Comput. Math. Appl. 59, 149-160 (2010)

21. Combettes, PL: The foundations of set theoretic estimation. Proc. IEEE 81, 182-208 (1993)

22. Alber, Yl, Guerre-Delabriere, S: Principles of weakly contractive maps in Hilbert spaces. Oper. Theory, Adv. Appl. 98 7-22 (1997)

23. Zhang, SS, Yang, L, Liu, JA: Strong convergence theorems for nonexpansive mappings in Banach spaces. Appl. Math. Mech. 28, 1287-1297 (2007)

24. Takahashi, W: Nonlinear Functional Analysis. Fixed Point Theory and Its Applications. Yokohama Publishers, Yokohama (2000)

25. Yaoa, Y, Lioub, Y-C, Chena, R: A general iterative method for an infinite family of nonexpansive mappings. Nonlinear Anal. 69, 1644-1654 (2008)

10.1186/1687-1812-2014-225

Cite this article as: Piri and Kumam: Strong convergence theorems for fixed points of asymptotically nonexpansive semigroups in Banach spaces. Fixed Point Theory and Applications 2014, 2014:225 